\title{
ESTADO ACTUAL DEL BANCO DE GERMOPLASMA DE PEJIBAYE (Bactris gasipaes), GUÁPILES, COSTA RICA ${ }^{1}$
}

\author{
Luis Diego Ríos-Reyes ${ }^{2}$,Elena Castillo-Hernández 2, Eric J.Fuchs-Castillo ${ }^{3}$
}

\section{RESUMEN}

Estado actual del banco de germoplasma de pejibaye (Bactris gasipaes), Guápiles, Costa Rica. El objetivo del presente estudio fue determinar el estado actual del banco de germoplasma de pejibaye en la Estación Experimental Los Diamantes (BGPLD). Se cuantificaron las accesiones perdidas y se obtuvo la información faltante en las boletas pasaporte para las accesiones sobrevivientes. Los datos de pasaporte se obtuvieron en parte del diario elaborado por Jorge Mora Urpí, fundador del BGPLD en el año 1972. Para aquellas accesiones sin datos se utilizaron fuentes bibliográficas para complementar la información. Se realizó un censo en el año 2013 para determinar la cantidad de palmeras en pie. El banco estaba originalmente compuesto por 7286 palmeras representando 837 accesiones de nueve países. Se identificaron 1471 individuos pertenecientes al grupo occidental y 1271 al grupo oriental. Actualmente solo sobreviven 805 individuos $(11,1 \%)$. Se observó una menor mortalidad en las palmeras del grupo occidental $(62,2 \pm$ $41,5 \%)$ que en el grupo oriental $(81,5 \pm 19,6 \%)$. El BGPLD se encuentra en un estado crítico de deterioro. Se deben llevar a cabo políticas concretas y urgentes para rescatar este importante recurso fitogenético.

Palabras clave: chontaduro, pupunha, pijuayo, manejo bancos de germoplasma, recursos genéticos.

\begin{abstract}
Current status of the peach palm (Bactris gasipaes) germoplasm bank, Guapiles, Costa Rica. The objective of this study was to determine the current status of the peach palm germoplasm bank at Los Diamantes Experimental Station (BGPLD). Lost accessions were quantified and missing passport data for the remaining accessions was obtained. The passport data was obtained from the diary created by Jorge Mora Urpí, founder of the bank, in 1972. To complete the information of accessions without data, bibliographic resources were used. In 2013, a census was conducted to determine the number of surviving palm trees. Originally, the bank was composed of 7286 palms representing 837 accessions from nine countries. A total of 1471 individuals belonging to the western group and 1271 to the eastern group were identified. Currently, only 805 individuals survive (11.1\%). A lower mortality was observed in the palms from the western group $(62.2 \pm 41.5 \%)$ compared to those in the eastern group $(81.5 \pm 19.6 \%)$. The BGPLD is in a critical condition of deterioration. Urgent and concrete institutional policies are needed to rescue this important plant genetic resource.
\end{abstract}

Keywords: chontaduro, pupunha, pijuayo, germplasm bank management, genetic resources.

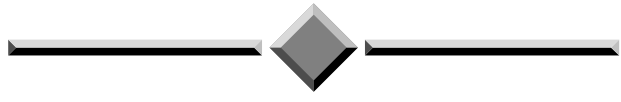

1 Recibido: 13 agosto, 2015. Aceptado: 15 de diciembre, 2015. Este trabajo forma parte del proyecto de investigación 111-B1-056 inscrito en la Vicerrectoría de Investigación de la Universidad de Costa Rica (UCR).

2 Universidad de Costa Rica, Escuela de Biología, San Pedro de Montes de Oca, San José, Costa Rica. Código postal 11501-2060.1uis.rios@ ucr.ac.cr, eric.fuchs@ucr.ac.cr (autor para correspondencia).

3 Universidad de Costa Rica, Centro de Investigaciones en Biología Celular y Molecular. Sede Regional del Atlántico, Turrialba, Cartago, Costa Rica.castillo.hernandez@gmail.com 


\section{INTRODUCCIÓN}

El pejibaye (Bactris gasipaes Kunth., Arecaceae) es la palmera de origen americano de mayor importancia económica (Mora-Urpí et al., 1997). Posee usos diversos, tales como hortaliza (palmito), fuente de frutos comestibles, aceite y material de construcción (Mora-Urpí et al., 1997). En la actualidad, Brasil, Colombia, Costa Rica y Perú son los mayores productores; sin embargo, entre un $40 \%$ y $50 \%$ de la producción se pierde o se utiliza como alimento animal (Clement et al., 2004). En Costa Rica, para el año 2013, la exportación de productos derivados de pejibaye generó ganancias por más de US\$ 17.5 millones (PROCOMER, 2014). Si se asume que solo se comercializó la mitad del producto, el potencial económico del pejibaye puede alcanzar hasta los US\$ 35 millones en ganancia.

El alto potencial económico del pejibaye se debe a su alto valor nutricional, su adaptación a climas tropicales húmedos (entre 2000-5000 mm lluvia, $>24^{\circ} \mathrm{C}$ ) y su amplia variabilidad morfológica (Graefe et al., 2012). Los frutos de pejibaye representan una fuente importante de vitamina $\mathrm{A}, \beta$-caroteno, potasio y carbohidratos, aunque contienen bajos niveles de proteínas y otros minerales (Leterme et al., 2005); el palmito exhibe una moderada cantidad de fibra, magnesio y hierro (Asunción, 1991). Por otro lado, la productividad de pejibaye varía entre las tres y veinte toneladas/ha/año en el caribe costarricense y el pacífico colombiano, respectivamente (Clement y Mora-Urpí, 1987; MADR 2009).

Según su sitio de origen, las palmeras de $B$. gasipaes varían en la presencia de espinas, así como en el tamaño y características del fruto (Mora-Urpí et al., 1997). El pejibaye se distribuye naturalmente desde Honduras hasta el centro de Bolivia y el estado de Pará en Brasil (Graefe et al., 2012). Según sus rasgos fenotípicos y relaciones genéticas, las poblaciones cultivadas se dividen en el grupo occidental (América Central y los valles inter-Andinos colombianos) y dos grupos orientales (Alto Amazonas y Amazonas Oriental) (Hernández-Ugalde et al., 2010). El grupo occidental se caracteriza por presentar tallos comparativamente más duros y hojas más grandes que el grupo oriental, así como muchas espinas y enraizamiento más sólido de los juveniles (Mora-Urpí et al., 1997).
Gran parte de esta variación morfológica y genética responde a procesos de selección artificial y natural (Tanksley y McCouch, 1997). Esta variabilidad es importante para el mejoramiento de la especie (FAO, 2014). A través del entrecruzamiento de plantas de distintas regiones se pueden producir variedades resistentes a patógenos o a cambios climáticos, así como variedades con una mayor productividad (Esquinas-Alcázar, 2005). Específicamente para $B$. gasipaes, el mejoramiento genético busca que las nuevas variedades para fruta presenten una ausencia de espinas en el tallo, hojas con una alta tasa de asimilación de carbono, madurez temprana ( $<3$ años), más de 100 frutos/racimo y frutos de más de $50 \mathrm{~g}$, entre otras (Mora-Urpí et al., 1997).

En pejibaye el mejoramiento genético depende de bancos de germoplasma. Estos almacenan razas o variedades de especies de interés comercial con semillas recalcitrantes (Brown et al., 1989). El potencial económico del pejibaye impulsó la creación de bancos de germoplasma en países como Brasil, Colombia, Costa Rica, Ecuador y Perú (Clement et al., 2004). La mayoría de estas colecciones resguardaban razas locales y pocas colecciones de otros sitios. Por ejemplo, de los diecisiete bancos que enlistan MoraUrpí et al. (1997), solo cuatro albergaban accesiones de más de cinco países. Entre ellos destaca el Banco de Germoplasma de Pejibaye en la Estación Experimental Los Diamantes (BGPLD) en Guápiles, Costa Rica, con 1200 accesiones de nueve países (Mora-Urpí et al., 1997).

El BGPLD se fundó en el año 1972 mediante el esfuerzo de Jorge Mora Urpí (Mora-Urpí, 1978). La colección funcionó como un sitio importante de investigación en pejibaye por más de treinta años (García, 2009) y Mora-Urpí logró producir nuevas variedades, siendo la más destacada Diamantes-10, la cual no tiene espinas y presenta un rendimiento comparativamente superior (Arroyo y Mora 2002; Bogantes et al., 2004), al igual que un palmito de alta calidad (Mora-Urpí et al., 1997; Bogantes et al., 2004; Clement et al., 2004).

En los últimos once años, el BGPLD ha sufrido un continuo deterioro por falta de políticas claras de conservación, mantenimiento y programas de recuperación. Esto ha empeorado desde el fallecimiento de su fundador en el año 2008. El objetivo del presente estudio fue determinar el estado actual del banco de 
germoplasma de pejibaye en la Estación Experimental Los Diamantes (BGPLD).

\section{MATERIALES Y MÉTODOS}

\section{Sitio de estudio}

El BGPLD se encuentra en la Estación Experimental Los Diamantes, la cual pertenece al Instituto Nacional de Innovación y Transferencia en Tecnología Agropecuaria (INTA). El sitio se ubica en Guápiles, cantón de Pococí, provincia de Limón $\left(10^{\circ} 13^{\prime} \mathrm{N}, 86^{\circ} 46^{\prime} \mathrm{O}\right)$ a $249 \mathrm{msnm}$. La estación posee 718 ha dedicadas a producción agrícola (INTA, 2014), de las cuales 60 ha corresponden al BGPLD. El sitio presenta una temperatura promedio anual de $27,5^{\circ} \mathrm{C}$ y una precipitación anual de $4500 \mathrm{~mm}$ (Bogantes et al., 2004).

\section{Validación de datos de pasaporte y mortalidad de} accesiones

Para determinar el número de accesiones vivas en pie se determinó la identidad de las palmeras a través de una revisión documental de fuentes primarias. Inicialmente se analizó la información presente en el diario del banco de germoplasma del Dr. Jorge MoraUrpí (1978); sin embargo, el diario no presentaba información completa de todas las accesiones, por lo que se actualizó mediante una revisión bibliográfica (Mattos-Silva 1992) y la información presente en el Herbario de la Universidad de Costa Rica (USJ). Para cada accesión se registró la identidad, el pasaporte, el país de origen, la provincia o departamento, la localidad de recolecta, así como la sección y parcela del banco en que se encontraba el individuo.

Se recopilaron datos de mortalidad para los años 1987, 2003 y 2013 para cada una de las accesiones con el propósito de describir el estado del BGPLD. Los dos primeros censos fueron realizados por el profesor Mora-Urpí (Récord de introducciones plantadas en el campo, Mora-Urpí, 1978). En el 2013 se realizó un censo de campo para obtener una estimativa de mortalidad, en los cuales se cuantificó la presencia o ausencia de cada individuo. De manera complementaria, para las secciones L, S, X, Y (MoraUrpí, 1978) se cuantificaron las palmeras con hijuelos que permitirían la recuperación del banco mediante reproducción vegetativa (Rengifo, 1993; Flores et al., 2012); esto con el fin de determinar el potencial de las accesiones para propagación. Se determinó el número de accesiones actuales y la tasa de mortalidad. Los resultados se presentan como el porcentaje de mortalidad entre censos y mortalidad acumulada según los grupos y razas propuestos por Mora-Urpí et al. (1993) y Hernández-Ugalde et al. (2010) para $B$. gasipaes.

\section{RESULTADOS Y DISCUSIÓN}

Según la revisión bibliográfica, el banco estaba originalmente compuesto de 7286 palmeras representado 837 accesiones (Mora-Urpí, 1978; MattosSilva, 1992). Se identificó con certeza el país de origen de un $74,8 \%$ de los individuos $(\mathrm{n}=5447)$ (Cuadro 1) y la raza de un $50 \%(n=3642)$ (Cuadro 2). Los países con más accesiones fueron Costa Rica, Brasil y Perú; mientras que Ecuador, Nicaragua y Venezuela fueron los países con menor número de representantes (Cuadro 1). Cuando se contrastaron los datos originales con el censo del 2013, del total de más de 7000 palmeras sembradas inicialmente en el banco de germoplasma solo sobreviven un $11,1 \%$ ( $\mathrm{n}=805$ individuos), de las cuales 142 presentaron hijuelos (Cuadro 1).

\section{Análisis por país y raza}

A través de la revisión bibliográfica se identificó que 1471 individuos pertenecían al grupo occidental y 2171 al grupo oriental (Cuadro 2). Ambos grupos evidenciaron una mortalidad comparable entre los censos de 1987 y 2003. La mortalidad promedio del grupo occidental fue de $13,3 \pm 11,9 \%$, mientras que en el grupo oriental fue $26,6 \pm 14,8 \%$. De manera similar, para el periodo 2003-2013 se observó un incremento significativo en la mortalidad de ambos grupos (62,2 \pm $41,5 \%$ vs. $81,5 \pm 19,6 \%$ ). Para el año 2013 solamente permanecían un $10,2 \%$ de las palmeras provenientes del grupo occidental, un $11,8 \%$ del subgrupo Alto Amazonas y un 3,0\% del grupo Amazonas Oriente (Cuadro 2).

Para el censo del 2013, las razas con mayor prevalencia fueron aquellas pertenecientes a Guatuso, Putumayo y Tuira (Cuadro 2). En contraparte, las 
Cuadro 1. Cantidad de accesiones de pejibaye (Bactris gasipaes, Palmae), según número de sobrevivientes al censo del 2013, individuos con hijuelos y país de origen originales en el banco de germoplasma en la Estación Experimental Los Diamantes, Guápiles, Costa Rica. 2013.

Table 1. Number of surviving peach palm (Bactris gasipaes, Palmae) accessions based on the number of surviving individuals from the 2013 census, number of individuals with offshoots and country of origin in the germplasm bank at Los Diamantes Experimental Station, Guápiles, Costa Rica, 2013.

\begin{tabular}{lccc}
\hline \multirow{2}{*}{ País } & \multicolumn{3}{c}{ Individuos (n) $^{n}$} \\
\cline { 2 - 4 } & Original & $\mathbf{2 0 1 3}^{\mathbf{a}}$ & Hijuelos $^{\mathbf{a}}$ \\
\hline Bolivia & 233 & $0(100)$ & $0(0)$ \\
Brasil & 1155 & $50(95,7)$ & $1(0,02)$ \\
Colombia & 778 & $129(83,4)$ & $24(18,6)$ \\
Costa Rica & 1611 & $308(80,9)$ & $53(17,2)$ \\
Ecuador & 230 & $8(96,5)$ & $0(0)$ \\
Nicaragua & 3 & $0(100)$ & $0(0)$ \\
Panamá & 308 & $92(70,1)$ & $5(5,4)$ \\
Perú & 1059 & $78(92,6)$ & $37(47,4)$ \\
Venezuela & 70 & $21(70)$ & $1(4,7)$ \\
Sin identificar & 1839 & $119(93,5)$ & $22(18,5)$ \\
Total & 7286 & $805(89)$ & $142(17,6)$ \\
\hline
\end{tabular}

a entre paréntesis se muestran los porcentajes de mortalidad/ mortalities percentage are shown in parenthesis.

otras siete razas identificadas presentaron menos de 31 individuos cada una. Las razas Cauca y Vaupés presentaron la menor cantidad de individuos (cinco y tres, respectivamente); mientras que todas las accesiones de la raza Tembé de Bolivia, desaparecieron.

Los resultados obtenidos demuestran que el BGPLD se ha deteriorado progresiva $y$ significativamente durante los últimos diez años. Para el año 2013, persistían aproximadamente solo una de cada diez palmeras sembradas originalmente en el banco. Estos resultados se asemejan a lo sucedido en otros bancos de germoplasma de pejibaye en el Neotrópico (Clement et al., 2004; de Cristo-Araújo et al., 2015). Se encontró una mayor tasa de mortalidad para las palmeras pertenecientes al grupo oriental que para las palmeras del grupo occidental. Estos resultados eran esperables, ya que las poblaciones de este último grupo provienen de sitios geográficamente cercanos al banco, por lo que probablemente están mejor adaptadas a las condiciones climáticas y edáficas del banco (Mora-Urpí et al., 1997; Graefe et al., 2012). Esto sugiere que las accesiones pertenecientes al grupo oriental debieron de ser monitoreadas para determinar si requerían cuidados particulares.

Se recomienda utilizar bancos de germoplasma descentralizados, es decir, colocar las accesiones en sitios con condiciones agro-ecológicas similares al sitio de donde son originarias (FAO, 2014). Estas pueden ser simuladas a través de cambios en la disponibilidad de agua, en la intensidad de sombra, en el tipo de suelo o a través de procesos agroquímicos (FAO, 2014). La Estación Experimental Los Diamantes, presenta suelos ácidos (Valverde, 1992) y una precipitación anual media alta (Bogantes et al., 2004). En teoría, estas condiciones son adecuadas para el desarrollo del cultivo (Mora-Urpí et al., 1997), por lo que es probable que la mayor mortalidad del grupo oriental se deba a requerimientos nutricionales específicos que no se lograron mantener.

Se lograron identificar 837 accesiones a partir de las fuentes primarias; sin embargo, Mora-Urpí et al. (1997) y García (2009) indicaron que el banco de germoplasma presentaba 1207 y 2000 accesiones, respectivamente. Esto ejemplifica la importancia de tener datos de pasaporte validados por curadores y en bases de acceso público (FAO, 2014), lo que evitaría que este tipo de contradicciones se repitan en el futuro. Asimismo, la mayoría de datos de pasaporte tenían información morfológica incompleta (Ríos, observación personal), por lo que sería necesario completar la descripción de cada pasaporte para cumplir con los estándares establecidos por la FAO (2014).

Un banco de germoplasma vivo requiere de un área extensa de siembra, equipo para caracterización de los individuos, transporte del material, control de plagas, aplicación de fertilizantes y vigilancia constante (FAO, 2014). Cada uno de estos aspectos implica una inversión significativa de recursos. Para iniciar y mantener un proyecto de este tipo durante un periodo prolongado, es necesario contar con apoyo financiero seguro (van Leeuwen et al., 2005). Por ejemplo, se estima que en el banco de germoplasma de B. gasipaes, del Instituto Nacional de Investigación del Amazonas (INPA, por sus siglas en portugués) en Manaus, Brasil, se invirtió un total de US\$ 177.500 a 
Cuadro 2. Mortalidad de las palmeras de pejibaye (B. gasipaes) del banco de germoplasma en la Estación Experimental Los Diamantes, Guápiles, Costa Rica. 2013.

Table 2. Mortality of the peach palms (B. gasipaes) from the germplasm bank at Los Diamantes Experimental Station, Guápiles, Costa Rica.

\begin{tabular}{|c|c|c|c|c|c|c|c|}
\hline \multirow{2}{*}{$\begin{array}{l}\text { Grupo } \\
\text { Subrupo }\end{array}$} & \multirow[t]{2}{*}{ Origen } & \multirow[t]{2}{*}{ Raza } & \multirow{2}{*}{$\begin{array}{c}\text { Año de } \\
\text { siembra }\end{array}$} & \multirow[t]{2}{*}{$\mathbf{N}$} & \multicolumn{3}{|c|}{ Censo } \\
\hline & & & & & 1987 & 2003 & 2013 \\
\hline \multirow[t]{5}{*}{ Occidental } & Alajuela, Costa Rica & Guatuso & 1977 & 670 & $308(45,2,-)^{a}$ & $254(17,5 ; 62,1)$ & $253(0,3,62,2)$ \\
\hline & Cartago, Costa Rica & Tucurrique & 1978 & 161 & $104(33,5,-)$ & $75(27,9 ; 53,4)$ & $12(84,92,5)$ \\
\hline & Darién, Panamá & Tuira & 1987 & 171 & - & $139(19,-)$ & $85(39,50)$ \\
\hline & Veraguas, Panamá & Azuero & 1978 & 128 & $63(50,8,-)$ & $62(1,9 ; 51,6)$ & $7(88,7,94,5)$ \\
\hline & $\begin{array}{l}\text { Valle del Cauca, } \\
\text { Colombia }\end{array}$ & Cauca & 1978 & 341 & $305(10,6,-)$ & $304(0,3 ; 10,9)$ & $3(99,99,1)$ \\
\hline \multicolumn{8}{|l|}{ Oriental } \\
\hline \multirow[t]{4}{*}{ Alto Amazonas } & Putumayo, Colombia & Putumayo & $?$ & 323 & - & - & $119(-, 63)$ \\
\hline & $\begin{array}{l}\text { Guaviare-Vaupés, } \\
\text { Colombia }\end{array}$ & Vaupés & 1987 & 18 & - & $10(44,-)$ & $5(50,72)$ \\
\hline & Loreto, Perú & Yurimaguas & 1978 & 510 & $365(28,4,-)$ & $324(11,2,36,5)$ & $54(76,3,89,4)$ \\
\hline & Amazonas, Brasil & Solimões & 1978 & 924 & $509(44,9,-)$ & $453(11,51)$ & $31(93,2,96,6)$ \\
\hline \multirow[t]{2}{*}{ Amazonas Oriente } & Pará, Brasil & Pará & $1980^{\mathrm{b}}$ & 162 & - & $100(34,-)$ & $12(88,92)$ \\
\hline & Cochabamba, Bolivia & Tembe & 1981 & 234 & $171(27,-)$ & $156(33,46)$ & $0(100,100)$ \\
\hline Otros sitios $^{c}$ & - & - & - & 582 & - & - & $71(-, 87,8)$ \\
\hline
\end{tabular}

aLos valores en paréntesis representan el porcentaje de mortalidad entre censos y la mortalidad acumulada, respectivamente / Values in parenthesis represent the percentage of mortality between censuses and the cumulative mortality, respectively.

b Para 1980, solo 90 palmeras habían sido sembradas, de las cuales diez murieron. En 1987 se sembraron 72 palmas adicionales. Las estimativas de mortalidad se basan en estas 152 palmeras / By 1980, only 90 palms had been planted, ten of which died. In 1987, 72 additional palms were planted. Mortality estimates are based on these 152 palms.

c Individuos para los cuales se obtuvo información geográfica adicional al país de origen. Incluye accesiones de Colombia (Cundinamarca), Costa Rica (Barra del Colorado, Buenos Aires, Bri Bri, Coto Brus, Guápiles, Siquirres, Pérez Zeledón, Tabarcia), Ecuador (Napo, Pastaza, Orellana, Sucumbios) y Venezuela (Mérida [Bactris macana], Zulia) / Individuals for which geographic information besides country of origin was found. These include accessions from Colombia (Cundinamarca), Costa Rica (Barra del Colorado, Buenos Aires, Bri Bri, Coto Brus, Guápiles, Siquirres, Pérez Zeledón, Tabarcia), Ecuador (Napo, Pastaza, Orellana, Sucumbios), and Venezuela (Mérida [Bactris macana], Zulia).

Los datos se presentan como el número de individuos presentes en cada censo según los grupos, subgrupos y razas de B. gasipaes / Data is presented as the number of individuals present tin each census according to B. gasipaes groups, subgroups, and races.

lo largo de dos décadas (van Leeuwen et al., 2005). En lo que respecta al BGPLD, aparte de la inversión para el mantenimiento, también se debería invertir en recuperar las accesiones en peligro. La mayoría de palmeras sobrevivientes son de más de $30 \mathrm{~m}$ de altura, etapa en que la mortalidad es más alta (Mora-Urpí et al., 1997), por lo que sería necesario podar algunas de las accesiones y propagarlas vegetativamente (por hijuelos o in vitro), así como fomentar esfuerzos de almacenar tejidos y ADN como los iniciados por el banco de germoplasma de pejibaye in vitro que se desarrolla en el Centro de Investigaciones Agronómicas (CIA) de la Universidad de Costa Rica.

La viabilidad de los bancos de germoplasma es vulnerada cuando el flujo de inversión se interrumpe y hay carencia de planes de desarrollo a mediano y largo plazo. Particularmente, en la Estación Experimental Los Diamantes, durante la década de los 2000 la investigación en pejibaye mermó en relación con décadas anteriores, lo que se reflejó en la cantidad de recursos asignados para el mantenimiento del banco y, por lo tanto, en la cantidad de individuos que 
sobrevivieron. Los bancos de germoplasma de plantas poco utilizadas deberían de ser de un tamaño modesto y enfocados en la demanda de producto (Clement et al., 2004). Esto conlleva un menor requerimiento de dinero y mayor utilización de las accesiones disponibles.

En la actualidad los bancos de $B$. gasipaes están sustituyéndose por colecciones núcleo que requieren menos recursos para su mantenimiento (de CristoAraújo, 2015). Estas colecciones consisten en un conjunto de accesiones que constituyen al menos el $70 \%$ de la diversidad genética de toda la colección original; con la menor repetición de accesiones posible (de Cristo-Araújo et al., 2015). Sería recomendable transformar el banco de germoplasma de pejibaye en la Estación Experimental Los Diamantes en una Colección Núcleo, de esta manera ocuparía menos espacio, se le podría dar el cuidado necesario a los pocos individuos sobrevivientes, lo cual se traduciría en más recursos para la investigación y la producción.

A corto plazo es imperativo rescatar el banco actual, desarrollar políticas de carácter institucional acordes con este objetivo y preservar a largo plazo este importante recurso fitogenético. Particularmente, es urgente tomar tres acciones para recuperar el BGPLD: i) formar alianzas público-privadas que permitan enlazar la investigación académica con la producción agrícola; ii) alcanzar un acuerdo entre las instituciones involucradas (Universidad de Costa Rica e Instituto Nacional de Innovación y Transferencia en Tecnología Agropecuaria), donde se definan las responsabilidades de ambas para la conservación del recurso; y iii) desarrollar una estrategia agresiva de recuperación y mantenimiento del banco. De lograrse, sería más asequible materializar la promesa del BGPLD de incrementar el consumo de productos nativos, de alto valor nutricional y con amplia diversidad de usos.

\section{AGRADECIMIENTOS}

Al personal de la Estación Experimental Los Diamantes por conceder permiso para realizar el trabajo de campo.A la Vicerrectoría de Investigación de la Universidad de Costa Rica por el apoyo financiero.

\section{LITERATURA CITADA}

Arroyo, C., y J. Mora. 2002. Producción comparativa de palmito entre cuatro variedades de pejibaye (Bactris gasipaes Kunth). Agron. Mesoam. 13:135-140.

Asunción, R. 1991. Caracterización química del palmito de pejibaye (Bactris gasipaes K.). Tesis Lic., Universidad de Costa Rica, San José, CRC.

Bogantes, A., R. Agüero, y J. Mora. 2004. Palmito de pejibaye (Bactris gasipaes K.): distancias de siembra y manejo de malezas. Agron. Mesoam. 15:185-192.

Brown, A.H.D., O.H. Frankel, D.R. Marshall, and J.T. Williams. 1989. The use of plant genetic resources. Press Syndicate of the University of Cambridge, Cambridge, GBR.

Clement, C.R., and J. Mora-Urpí. 1987. Pejibaye palm (Bactris gasipaes, Arecaceae): multi-use potential for the lowland humid tropics. Econ. Bot. 41:302-311.

Clement, C.R., J.C. Weber, J. van Leeuwen, C.A. Domian, D.M. Cole, L.A. Arévalo-López, and H. Argüello. 2004. Why extensive research and development did not promote use of peach palm fruit in Latin America. Agrofor. Syst. 61:195-206.

de Cristo-Araújo, M., D.P. Rodrigues, S. Astolfi-Filho, and C.R. Clement. 2015. Peach palm core collection in Brazilian Amazonia. CBAB 15:18-25.

Flores, W.B.C., K. Yuyuma, and R.G. da Silva. 2012. Asexual propagation of peach palm by division of the clump and extraction of the off-shoots. Hortic. Bras. 30:151-154.

García, J. 2009. Jorge Mora Urpí (12 julio 1930 - 28 octubre 2008) Un nombre asociado a la biología del pejibaye (Bactris gasipaes H.B.K.). Rev. Biol. Trop. 57:XIX - XXIII.

Graefe, S., D. Dufour, M. van Zonneveld, F. Rodriguez, and A. González. 2012. Peach palm (Bactris gasipaes) in tropical Latin America: implications for biodiversity conservation, natural resource management and human nutrition. Biodivers. Conserv. 22:269-300.

Esquinas-Alcazar, J. 2005. Protecting crop genetic diversity for food security: political, ethical and technical challenges. Nature 6:946-953.

FAO. 2014. Genebank standards for plant genetic resources for food and agriculture. FAO, Roma, ITA.

Hernández-Ugalde, J., J. Mora-Urpí, and O.J. Rocha. 2010. Genetic relationships among wild and cultivated 
populations of peach palm (Bactris gasipaes Kunth, Palmae): evidence for multiple independent domestication events. Genet. Resour. Crop Evol. 58:571-583.

INTA (Instituto Nacional de Innovación y Transferencia en Tecnología Agropecuaria). 2014. Estación Experimental Los Diamantes. http://www.inta.go.cr/ estaciones-experimentales/los-diamantes (consultado 28 jun. 2014)

Leterme, P., M.F. García, A.M. Londonño, M.G. Rojas, A. Buldgen, and W.B. Souffrant. 2005. Chemical composition and nutritive value of peach palm (Bactris gasipaes Kunth) in rats. J. Sci. Food Agric. 85:1505-1512.

MADR (Ministerio de Agricultura y Desarrollo Rural). 2009. Anuario estadístico de frutas y hortalizas 2004 2008. MADR, Bogotá, COL.

Mattos-Silva, L. 1992. Diferenciación taxonómica de diez razas de Pejibaye cultivado (Bactris (guilielma) gasipaes Kunth.) y su relación con otras especies de Bactris. Tesis MSc., Universidad de Costa Rica, San José, CRC.

Mora Urpí, J. 1978. Pejibaye (Bactris gasipaes H.B.K.). Banco de germoplasma. Récord de introducciones plantadas en el campo. Fecha de inicio: enero 11 de 1978. Campo situado en la Estación Experimental "Los Diamantes". Programa de Pejibaye - Jorge Mora Urpí. N ${ }^{\circ}$ 1. UCR-MAG-Asbana, Limón, CRC.

Mora-Urpí, J., C. Clement, and V. Patiño. 1993. Diversidad Genética en Pejibaye: II. Origen y Evolución. En: J. Mora-Urpí et al., editores, IV Congreso Internacional sobre biología, agronomía e industrialización del pijuayo, Iquitos, Perú. Universidad de Costa Rica, San José, Costa Rica. p. 21-29.

Mora-Urpí, J., J.C. Weber, and C.R. Clement. 1997. Peach palm, Bactris gasipaes Kunth. Promoting the conservation and use of underutilized and neglected crops. 20. Institute of Plant Genetics and Crop Plant Research, Gatersleben / International Plant Genetic Resources Institute, Roma, ITA.

PROCOMER (Promotora de Comercio Exterior de Costa Rica). 2014. Portal estadístico de comercio exterior. PROCOMER. http://servicios.procomer.go.cr/ estadisticas/inicio.aspx (consultado 18 jul. 2015).

Rengifo, D.P. 1993. Propagación vegetativa de Bactris gasipaes Bailey (pijuayo). Folia Amazónica 5:16-37.

Tanksley, S.D., and S.R. McCouch. 1997. Seed banks and molecular maps: unlocking genetic potential from the wild. Science 277:1063-1066.

Valverde, L.G. 1992. Estudio detallado de suelos de la Finca Experimental Los Diamantes. Atlantic Zone Programme, Centro Agronómico Tropical de Investigación y Educación (CATIE), Agricultural University Wageningen (AUW), Ministerio de Agricultura y Ganadería (MAG), CRC.

van Leeuwen, J., E. Lleras-Pérez, and C.R. Clement. 2005. Field genebanks may impede instead of promote crop development: lessons of failed genebanks of “promising” Brazilian Palms. Agrociencia 9:61-66. 
